Rotavirus is the main cause of AGE accounting for about $35 \%$ of all causative agents.

About $90 \%$ of all cases of Rotavirus AGE occur in children under 5 years of age and

More than $50 \%$ of cases occur in infants.

Vaccination against rotavirus is now the best preventive measure.

\section{CASE REPORT - A PATIENT WITH BONE FORMATION DEFECTS DURING PRENATAL LIFE DIAGNOSED AS 'SHWACHMAN-DIAMOND SYNDROME'}

doi:10.1136/archdischild-2012-302724.0703

${ }^{1} \mathrm{O}$ Beser, ${ }^{2} \mathrm{D}$ Dokurel, ${ }^{1} \mathrm{~T}$ Kutlu, ${ }^{1} \mathrm{~T}$ Erkan, ${ }^{1} \mathrm{FC}$ Cokugras. ${ }^{1}$ Pediatric Gastroenterology, Hepatology and Nutrition; ${ }^{2} D e p t$. of Pediatrics, Istanbul University Cerrahpasa Medical Faculty, Istanbul, Turkey

CaseReport: Shwachman-Diamond syndrome(SDS) is an autosomal recessive disorder with clinical features that include pancreatic exocrine insufficiency, hematological dysfunction and skeletal abnormalities during development after birth and varies with age. The patient was born as the first child to healty, term. Labour was induced at $20 \mathrm{wk}$ gestation because of symetrical fetal growth retardation. Congenital infections were excluded. In the fetal karyotype analysis, no chromosomal abnormalities were detected. Molecular genetic analysis for achondroplasia and hypocondroplasia was performed and gave negative results. After birth, laboratory studies were normal. At the age of six months the patient's height, weight and head circumference remained below 3 percentile. During the sixth month of life, the child's blood count were evaluated retrospectively and intermittent neutropenia was documented. When she presented with increased fat loss and failure to thrive, fecal elastase level was $<50 \mathrm{ug} / \mathrm{g}$, low serum trypsinogen level was found. These results were primarily the signs of severe pancreatic insufficiency. On the CT scanning of pancreas, scattered fatty infiltration was detected. The X-ray imaging of the thorax showed narrow thorax. Although on spinal X-ray metaphyseal changes on the costochondral junctions was not detected; 'Metaphyseal dysostosis' of the femoral head and distal femur the was observed more remarkable than upper limbs. When SBDS gene which is a causative gene for Scwachman-Diamond Syndrome was analyzed by DNA sequence analysis.

Discussion In contrast to the previous observations, results suggest that the characteristic skeletal changes present in some patients in the intrauterine period of life. Our observation underlines the necessity to consider SDS as the other common reasons of prenatally diagnosed bone disorders like achondroplasia and hypocondroplasia.

\section{MALROTATION - NOT A LAUGHING MATTER}

doi:10.1136/archdischild-2012-302724.0704

E Robertson, M Thorpe. Paediatrics and Child Health, Royal Cornwall Hospital, Truro, UK

Background Early diagnosis and management of intestinal malrotation can potentially prevent the devastating complications associated with midgut volvulus. Atypical presentation of intestinal malrotation in older children frequently leads to a significant delay in diagnosis.

Methods A 6-year-old boy presented with a five year history of hiccups, then vomiting after laughing. Sadly, he had resorted to avoiding laughter. Barium studies demonstrated intestinal malrotation, therefore a Ladd's procedure was performed and he made a good recovery. The case prompted a literature search for aetiology, diagnosis and management of malrotation in children outside of the neonatal period.

Results We present a review of this literature, including findings that diagnosis of intestinal malrotation in the older child is frequently delayed. One study demonstrated a mean delay to diagnosis of 1.7 years. Vague abdominal symptoms including chronic abdominal pain, intermittent non-bilious vomiting, diarrhoea and failure to thrive have been associated with malrotation, but hiccups and laughter have not previously been described. Recent review of diagnostic imaging recommended ultrasound as the investigation of choice, and may advocate its use in the work up of children presenting with non-specific abdominal symptoms. Although debated in the literature, a Ladd's procedure is recommended for all children, even if asymptomatic.

Conclusion Children with intestinal malrotation present us with a diagnostic challenge, but a low threshold of suspicion in patients presenting with vague abdominal symptoms, including intractable hiccups or vomiting, may ensure these children receive the timely surgical intervention.

\section{GASTROINTESTINAL HEMORRHAGE IN A 21-MONTH-OLD GIRL IN THE COURSE OF LYMPHOODULAR HYPERPLASIA. CASE REPORT}

doi:10.1136/archdischild-2012-302724.0705

A Mroczkowska-Juchkiewicz, A Pawłowska-Kamieniak, D Gołyska, K Kominek, E Pac-Kozuchowska. Medical University, Lublin, Poland

Gastrointestinal hemorrage is an emergency condition requiring immediate diagnostics. Lymphonodular hyperplasia, as a cause of bleeding from the gastrointestinal tract in children, is a rarely observed pathology of unknown etiology. The case of a 21-monthold girl is presented with massive bleeding from the lower gastrointestinal tract in the course of lymphonodular hyperplasia. Endoscopy of the upper and lower gastrointestinal tract was performed as an emergency procedure. Gastroscopic examination did not indicate any deviations from the normal. Colonoscopy showed no organic pathology of the mucosa in the terminal ileum, and the presence of blood in the small intestine was not observed. In the entire large intestine the presence of hemolyzed blood was noted and a massive lymphonodular hyperplasia on the mucosa. Histopathologic tests of the specimens taken from the large intestine confirmed normal pit patterns, mucosal edema, presence of lymph follicles, and numerous apoptic bodies in the lamina propria beneath the epithelium and in the surface epithelium.

\section{INTESTINAL EPITHELIAL DYSPLASIA - A CASE REPORT OF A MILDER PHENOTYPE}

doi:10.1136/archdischild-2012-302724.0706

${ }^{1} \mathrm{G}$ Lesanu, II Tincu, ${ }^{2} \mathrm{M}$ Gherghiceanu, ${ }^{1} \mathrm{C}$ Becheanu, ${ }^{3} \mathrm{M}$ Dumbrava, ${ }^{1 D}$ Pacurar, 'V Danila. 'Grigore Alexandrescu' Emergency Children's Hospital; 2'Victor Babes' National Institute; ${ }^{3}$ Fundeni' Clinical Institute, Bucharest, Romania

The intractable diarrhea of infancy remains a difficult challenge for the pediatrician.

We report a rare case of a five-month-old female infant admitted in "Grigore Alexandrescu" Emergency Children's Hospital, Bucharest for diarrheic stools and severe malnutrition with birth onset. She was exclusively breastfed with poor gain weight $(360 \mathrm{~g}$ in 5 months). During the 5 months of life the child was repeatedly admitted to other hospitals because of diarrhea and failure to thrive without a precise diagnosis. We mention that she had a sister who died at 3 months old because of severe diarrhea with birth onset. At admission the infant had severe malnutrition with watery diarrhea and facial dysmorphism. After extensive investigations we excluded infections and allergic causes, cystic fibrosis, immune and autoimmune enterophaty, motility disorders. We performed inferior and superior endoscopies with biopsies that excluded microvillus inclusion disease. Examination of the biopsy specimens (optic and electronic microscopy) revealed the diagnosis of intestinal epithelial 
dysplasia (IED). The patient received parenteral and enteral nutrition with elemental formulas.

Previous reports have suggested that IED may be a congenital inherited autosomal recessive disease. We report a case of congenital enteropathy that represents a diagnostic and therapeutically challenge.

\section{EOSINOPHILIC DIGESTIVE DISEASE AND ATOPIC BRONCHIAL ASTHMA; TWO DISEASES OR AN EXPRESSION OF ONE DISEASE IN TWO DIFFERENT SYSTEMS}

doi:10.1136/archdischild-2012-302724.0707

M Yakoot. Internal Medicine and Pediatrics, Green Clinic and Research Centre, Alexandria, Egypt

Eosinophilic digestive disease (EDD) includes a broad spectrum of clinical presentations due to eosinophilic inflammation involving anywhere from the esophagus to the rectum. The heterogeneity in the clinical presentations of EDD is determined by the site and depth of eosinophilic infiltration. The sites of inflammation determine the nomenclature for EDD. The most well characterized of these, eosinophilic esophagitis (EE), eosinophilic gastroenteritis (EG), and eosinophilic colitis or enterocolitis. While the depth of esosinophilic infiltration through the three main layers (mucosa, musculosa and serosa) determines the prominent clinical manifestation. The recent advances in gastrointestinal endoscopy and the increasing awareness and diagnosis of EDD, in my viewpoint, can be of help to add to our understanding of the heterogeneous clinical syndrome under the broad title bronchial asthma.

Here I present a multidisciplinary comparitive analysis to prove that EDD and the allergic bronchial asthma can be regarded as two clinical expressions of one disease in two systems that are functionally different but anatomicaly and embryologically related.

\section{PREVALENCE OF FAT-SOLUBLE VITAMIN DEFICIENCIES IN CHILDREN WITH CYSTIC FIBROSIS}

doi:10.1136/archdischild-2012-302724.0708

AK Gupta, V Ng, J Garside. Paediatrics, Calderdale and Huddersfield NHS Trust, Halifax, UK

Background Malabsorption of fat-soluble vitamins is likely in most patients with cystic fibrosis, particularly those who are pancreatic insufficient. Low vitamin levels are associated with poorer clinical status, and reduced lung function. Since the introduction of improved pancreatic enzymes, normal to high fat diets and routine vitamin supplementation, clinical evidence of fat-soluble vitamin deficiency is rarely seen.

Aim To determine the prevalence of fat-soluble vitamin deficiencies in children with cystic fibrosis in Calderdale and Huddersfield NHS Trust.

Method Retrospective analysis of vitamin levels performed in children with cystic fibrosis in Calderdale and Huddersfield Trust over a period of six years. Data was available for 19 out of 22 children with cystic fibrosis. Results of plasma vitamin A, D (Total $25 \mathrm{OH}$ Vit D) and $\mathrm{E}$ levels for these children were collected on a pre-designed proforma.

Results Vitamin A, D and E levels were checked for a total of 75, 75 and 76 times respectively over a period of six years. Vitamin E levels were normal. Vit A levels were subnormal on two occasions. However, Vitamin D levels were sub-optimal (<60nmol/1) on forty occasions and amongst them levels were below $20 \mathrm{nmol} / \mathrm{l}$ on three occasions.

Conclusion Sub-optimal Vitamin D levels are still very common in children with cystic fibrosis despite routine vitamin supplementation.

\section{SPREADING OF NOURISHMENT DISORDERS IN SCHOOLCHILDREN POPULATION}

doi:10.1136/archdischild-2012-302724.0709

${ }^{1} \mathrm{~T}$ Zerekidze, ${ }^{2} \mathrm{~N}$ Adamia, ${ }^{3} \mathrm{M}$ Surviladze, ${ }^{1}$ Chkhaidze, ${ }^{2} \mathrm{~N}$ Katamadze, ${ }^{3} \mathrm{M}$ Kartozia, ${ }^{4} \mathrm{D}$ Khachapuridze, ${ }^{3} \mathrm{~V}$ Kherkheulidze, ${ }^{1} \mathrm{M}$ Gugunishvili. ${ }^{\top}$ Tbilisi State Medical University; ${ }^{2}$ Department of General Pediatrics, Tbilisi State Medical University; ${ }^{3} \mathrm{M}$. Iashvili Pediatric Clinic, Tbilisi; ${ }^{4}$ Kutaisi Pedagogical University, Kutaisi, Georgia

Goal Study of nourishment disorders' spreading and evaluation of the impact of risk factors in schoolchildren population.

Materials and Methods Study included 3012 children of 6-15 age: 1654 girls and 1358 boys. By means of the questionnaire we studied prevalence of nourishment disorders among schoolchildren population; evaluated peculiarities of clinical course and mathematically evaluated frequency and combinations of risk-factors. Statistical processing was provided by SPSS/v.12 software.

Research results Study was conducted by stages. In $47.2 \%$ of cases set of gastroezophagical symptoms was identified. Parents of $41 \%$ of the questioned children had gastrointestinal pathologies. Together with routine studies, $\mathrm{pH}$-metry, measurement of amylase and lipase levels in blood and urine was conducted.

Studies showed various organic pathologies in $57 \%$ of children, among them: gastric and duodenal ulcer in $9 \%$ of cases, pancreatitis, reflex-esophagitis, associated with ulcer in 19\% of cases, diaphragm hernia in $8 \%$.

In $48 \%$ of cases organic pathologies, recurrent diseases, congenital disorders were excluded. Mentioned population had the diagnosis of functional dyspepsia, with the further relevant treatment scheme and diet.

Conclusion Data of our epidemiological studies do not significantly differ from the data of various countries and in our case the contributing factor is Georgian cuisine.

\section{MAGNETIC RESONANCE IMAGING VERSUS ULTRASONOGRAPHY IN ASSESSING CHANGES IN FAT LIVER CONTENT IN OBESE CHILDREN AFTER ONE-YEAR NUTRITIONAL INTERVENTION}

doi:10.1136/archdischild-2012-302724.0710

'E Verduci, ${ }^{2} \mathrm{C}$ Pozzato, 'G Radaelli, ${ }^{2} \mathrm{~A}$ Rovere, 'M Salvioni, 'G Banderali, 'E Riva, 'M Giovannini. 'Department of Pediatrics; ${ }^{2}$ Department of Radiology, University of Milan, Milan, Italy

Background and Aims Despite the potential clinical and practical relevance, there is lack of studies in current literature assessing the relationship of longitudinal change of liver fat content with liver biochemical parameters in paediatric age. The aim of the present study was to assess whether any association may exist of change in liver fat content based on MRI with change in liver biochemical parameters in obese children who underwent a one-year nutritional intervention.

Methods Fourthy six obese children, aged 6-14 years, underwent metabolic measurements, liver ultrasonography (US) and chemicalshift MRI examinations at baseline and after an one-year nutritional intervention. Biochemistry included serum alanine aminotransferase (ALT) and aspartate aminotransferase (AST). Liver fat fraction (FF) on MRI was judged elevated as it was $39 \%$.

Results Prevalence of FF3 $9 \%$ declined from $34.8 \%$ to $8.7 \%(P<0.01)$, with a mean $(95 \% \mathrm{CI})$ reduction of $7.8(5.0-10.6) \%$. At baseline, FF was associated with any liver biochemical parameters (maximum $P<0.001)$. At the end of intervention association was found with AST $(P=0.017)$. Change of FF was associated with change in AST $(P=0.027)$ and $\operatorname{ALT}(P=0.024)$. Liver echogenicity was associated with ALT at baseline $(P<0.001)$. An age and sex adjusted multiple regression analysis showed that FF change was independently associated with change in serum AST (adjusted regression coefficient 0.348, $P=0.048$ ). 